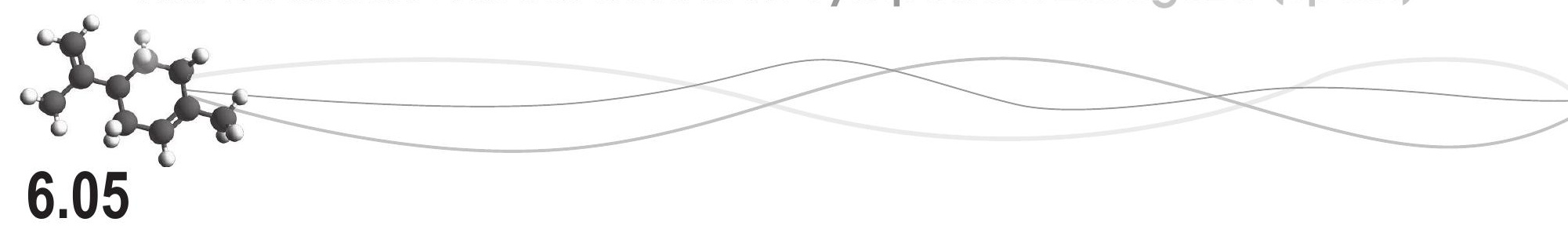

\title{
PTR-TOF-MS MONITORING OF IN-VITRO AND IN-VIVO FLAVOUR RELEASE IN CEREAL BARS WITH VARYING SUGAR COMPOSITION
}

\author{
Samuel Heenan ${ }^{2}$, Christos Soukoulis ${ }^{1}$, Patrick Silcock ${ }^{2}$, FRANCO BIASOLI, Eugenio \\ Aprea ${ }^{1}$, Luca Cappellin ${ }^{1}$, and Flavia Gasperi ${ }^{1}$ \\ ${ }^{1}$ IASMA Reseach and Innovation Centre, Fondazione Edmund Mach, Food Quality and \\ Nutrition Area, Via E. Mach, 1, 38010, S. Michele a/A, Italy \\ 2 Sensory Science Research Centre, Department of Food Science, University of Otago, \\ Dunedin, New Zealand \\ franco.biasioli@iasma.it
}

Keywords: PTR-TOF-MS; in-vivo, in-vitro, cereal bars, sweeteners; polydextrose

Proton transfer reaction mass spectrometry (PTR-MS) is a well-established technique used to assess volatile compounds release from real or model food matrices and, in particular, it has been applied to the in-vivo monitoring of flavour release during food consumption (nose space analysis) to demonstrate the influence of parameters related with the food matrix (composition, microstructure, textural and rheological properties) or the assessor's oral physiology e.g. saliva flow, swallowing, mouth volume and breathing pattern $(1,2)$. The recently developed PTR-ToF-MS combines the advantages of the quadrupole based PTR-MS (sensitivity, dynamic on-line capability) with improved mass and time resolution providing unambiguous determination of chemical formulas and the possibility to monitor complex matrixes without the necessity to select few compounds at a time (3).

Type and amount of sugars can modify the physical properties of food products influencing texture, appearance and possibly flavour release. Therefore changing sugar content, in view e.g. of sugar reduction, can influence the partitioning of aroma compounds, posing a critical issue for food manufacturers (4).

The present study has a twofold goal: firstly, to describe the application of the novel PTRToFMS in nose-space analysis and, secondly, to investigate by this technique the impact of changes in the level of glucose syrup substitution by polydextrose (from $0 \%$ to $70 \%$ on glucose syrup weight) both on the "in-vitro" and "in-vivo" flavour release from a complex system as flavoured cereal bars.

After describing the performances of PTR-ToF-MS we show how in the case of in-vitro analysis, the concentration of the flavor compounds was significantly affected by the sugar composition. The substitution of glucose syrup solids by polydextrose facilitated the release of acetaldehyde, esters, menthol and menthone whereas no significant effects were observed for other flavor compounds such as methyl cinnamate or vanillin. Flavor release is possibly driven by a "salting-out" effect due to the ability of polydextrose to enhance the rubbery glass transition state of the systems and consequently to reduce the water free volume.

In the case of in-vivo measurements, three parameters were calculated from the curves describing the nose-space concentration of each assessor: the total area $\left(\mathrm{A}_{\text {total }}\right)$, the maximum concentration $\left(\mathrm{I}_{\max }\right)$ and the time needed to reach it $\left(\mathrm{t}_{\max }\right)$. Moreover the time of the swallowing events has also been recorded. Similarly to the in-vitro analysis, esters, carboxylic 
acids, acetaldehyde, menthone and menthol exhibited significantly $(\mathrm{p}<0.05)$ higher values for $A_{\text {total }}, I_{\max }$, and $t_{\max }$ at a 50 or $75 \%$ glucose syrup substitution level. A similar mechanism related with salting out effects can be also used for explaining the observed behavior. For methyl cinnamate and vanillin $A_{\text {total }}$, and $I_{\max }$ were maximized at $75 \%$ glucose syrup substitution level but $t_{\max }$ seems to be related only to the swallowing time. This, together with the observed dependence of the swallowing behavior on sugar concentration, suggests that factors related with individual chewing behavior (free chewing protocol) and oral physiology may have led to the increased variability of the calculated parameters, particularly in the case of the time between the first swallowing event and $t_{\max }$.

References:

1. Buettner A.,\& Beauchamp J. (2010). Chemical input - sensory output: Diverse modes of physiology-flavour interaction. Food Quality and Preference, 21, 915-924

2. de Roos K.B. (2003). Effect of texture and microstructure on flavour retention and release. International Dairy Journal, 13, 593-605

3. Cappellin L., Biasioli F., Granitto P.M., Schuhfried E., Soukoulis C., Costa F., Märk T.D., and Gasperi F. (in press). On data analysis in PTR-TOF-MS: From raw spectra to data mining. Sensors and Actuators B: Chemical,doi:10.1016/j.snb.2010.11.044

4. Heenan S.P., Dufour J.P., Hamid N., Harvey W. \& Delahunty C.M., (2010). The influence of ingredients and time from baking on sensory quality and consumer freshness perceptions in a baked model cake system. LWT-Food Science and Technology, 43, 1032-1041 